\title{
Effect and mechanism of miR-126 in myocardial ischemia reperfusion
}

\author{
B. Li*, Y. Tao* and Q. Huang \\ Department of Cardiology, Jiangxi Provincial People's Hospital, Donghu District, \\ Nanchang, Jiangxi, China \\ *These authors contributed equally to this study. \\ Corresponding author: Q. Huang \\ E-mail: hellojimepp@163.com
}

Genet. Mol. Res. 14 (4): 18990-18998 (2015)

Received August 5, 2015

Accepted October 26, 2015

Published December 29, 2015

DOI http://dx.doi.org/10.4238/2015.December.29.6

ABSTRACT. Multiple studies have shown microRNAs to play an important role in disease occurrence and development. The role of miRNAs in ischemia-reperfusion injury, however, requires further investigation and the aim of this study was therefore to assess miR-126 expression in myocardial ischemia reperfusion and the effects of miR-126 on myocardial ischemia-reperfusion injury. An in vitro model of ischemia-reperfusion injury was established using rat myocardial $\mathrm{H} 9 \mathrm{c} 2$ cells and miR-126 expression in these cells was assessed by real-time PCR. The miR-126 mimic and inhibitor were transfected into $\mathrm{H} 9 \mathrm{c} 2$ cells before the injury was induced. Flow cytometry and western blotting were used to assess myocardial cell apoptosis. The triphenyltetrazolium chloride method was used to assess the infarction area and a TUNEL assay was used to analyze myocardial cell apoptosis. The results of the western blot analyses indicate that the miR126 mimic and inhibitor increase and decrease caspase 3 degradation in myocardial cells, respectively. The in vivo experiments, moreover, revealed that the miR-126 mimic and inhibitor increase and reduce the myocardial infarction area, respectively. The TUNEL assay results showed increases and decreases in apoptotic myocardial cell numbers after infusion with the miR-126 mimic or inhibitor, respectively. These findings indicate that miR- 
126 is down-regulated in myocardial ischemia-reperfusion injury and that the inhibition of miR-126 may protect against myocardial cell apoptosis caused by ischemia-reperfusion.

Key words: miR-126; Myocardial ischemia-reperfusion; Apoptosis

\section{INTRODUCTION}

In the treatment of ischemic cardiomyopathy, reperfusion therapy can reduce the myocardial necrosis area overtime and is currently the main treatment for acute myocardial infarction (Chen et al., 2013; Pirhan et al., 2013; Liu et al., 2014). Recent studies, however, have shown that sudden blood flow restoration in the coronary artery can cause myocardial reperfusion injury (Catalyurek et al., 2008; Yaghoubi et al., 2011; Medina et al., 2012). As much as 40-50\% of cardiac necrosis was recently shown to be caused by reperfusion injury (Alexandre et al., 2013; Cong et al., 2013; Qiao et al., 2013) and reducing reperfusion injury may therefore be effective in preventing and treating acute myocardial infarction.

The mechanism of myocardial ischemia-reperfusion injury remains unclear. Apoptosis has been proposed as one of the important causes of myocardial ischemia-reperfusion injury (Cayan et al., 2014; Hadi et al., 2014; Produit-Zengaffinen et al., 2014); however, the exact mechanism by which apoptosis is induced in this context still requires further investigation. It was recently shown that microRNAs (miRNAs) may play a major role in regulating myocardial ischemia reperfusion (Shapiro et al., 2011; Chang et al., 2012; Tu et al., 2013). miRNAs are small, endogenous, single-stranded, non-coding RNAs usually 22 nucleotides in length and function as regulators of protein expression by binding to mRNA and preventing translation (Liu et al., 2012; Quarles et al., 2013). The number of studies reporting on important roles for miRNAs in disease occurrence and development is steadily increasing and a number of studies have suggested a role for miRNAs in cardiovascular disease (Corsten et al., 2010). This potential association, however, requires further investigation. Studies on miR-126 have primarily been focused on malignant mesothelioma, where miR-126 has been shown to downregulate the malignancy (Tomasetti et al., 2014). miR-126 has also been shown to be involved in the PI3K signaling pathway (Guo et al., 2008). The role of miR126 in ischemia-reperfusion injury remained unclear and in this study, we therefore investigated and clarified the role of miR-126 in myocardial ischemia-reperfusion injury.

\section{MATERIAL AND METHODS}

\section{Cell culture}

Rat myocardial $\mathrm{H} 9 \mathrm{c} 2$ cells were purchased from ATCC. Cells were maintained in DMEM medium containing 10\% fetal bovine serum (Gibco, Carlsbad, CA, USA) and $100 \mu \mathrm{g} / \mathrm{mL}$ streptomycin (Gibco, Carlsbad, CA, USA) at $37^{\circ} \mathrm{C}$ and $5 \% \mathrm{CO}_{2}$.

\section{miRNA transfection}

miR-126 inhibitor and mimic (Dharmacon, Austin, TX, USA) were transfected into the myocardial cells according to the manufacturer's instructions. H9c2 cells $\left(1 \times 10^{6}\right)$ were transfected with $0.4 \mathrm{nM}$ miR-126 mimic or inhibitor, and the cells were collected after $48 \mathrm{~h}$. 


\section{In vitro ischemia-reperfusion (SI/R) model}

After $48 \mathrm{~h}$ of transfection, cells were confirmed to be an ischemia-reperfusion model according to the method described by Ekhterae and colleagues (Ekhterae et al., 1999). The cells were maintained in glucose-free and serum-free Dulbecco's Modified Eagle Medium (DMEM) (Gibco, Carlsbad, CA, USA) in a hypoxia incubator for $10 \mathrm{~h}$, and then cultured with DMEM containing $10 \%$ fetal bovine serum at normal oxygen levels for $2 \mathrm{~h}$ after $2 \mathrm{~h}$ of oxygen training.

\section{Real-time PCR}

Total RNA was extracted from cells using Trizol (Life Technologies, Carlsbad, CA, USA) and cDNA was synthesized from the RNA using a TaqMan MicroRNA Reverse Transcription kit (Applied Biosystems, Waltham, Massachusetts, USA). miR-126 levels were determined by a Taqman miRNA assay and a Taqman Universal PCR Master Mix kit (Applied Biosystems, Waltham, Massachusetts, USA).

\section{Western blotting}

Total protein was extracted using RIPA buffer (containing $150 \mathrm{mM} \mathrm{NaCl}, 1.0 \%$ IGEPAL ${ }^{\circledR}$ CA-630, 0.5\% sodium deoxycholate, 0.1\% SDS, $50 \mathrm{mM}$ Tris, pH 8.0) (Sigma-Aldrich, St. Louis, MO USA) and was separated on $10 \%$ SDS-PAGE and then transferred it to an NC membrane. The membrane was blotted with anti-caspase-3 antibody (1:1000 dilution) (Cell Signaling Technology, Danvers, MA, USA). Bound antibody was visualized using HRP-conjugated anti-rabbit secondary antibody and enhanced chemiluminescence.

\section{Flow cytometry}

$\mathrm{H} 9 \mathrm{c} 2$ cells were harvested from mice ischemia-reperfusion (SI/R) model and were suspended in $1 \mathrm{x}$ binding buffer. After that, Annexin V-FITC and propidium iodide (PI; BD Bioscience, San Jose, CA, USA) were added into the solution and incubated for $30 \mathrm{~min}$ at room temperature in dark followed by analysis by flow cytometry.

\section{Rat myocardial ischemia reperfusion model}

Female Wistar rats (12-16 weeks) were purchased from Shanghai SLAC laboratory animal Co., Ltd. All rats were housed in an SPF animal experiment center at $22^{\circ}-24^{\circ} \mathrm{C}$ with a $12 \mathrm{~h}$ daily rhythm and received food and water ad libitum. The rats were grouped as follows: control group $(\mathrm{N}=16)$; ischemia reperfusion group ( $N=16$, myocardial ischemia for 30 min and reperfusion for $2 \mathrm{~h})$; ischemia reperfusion + virus mock-vehicle group $(\mathrm{N}=16,7$ days after virus mock-vehicle transfection, myocardial ischemia for $30 \mathrm{~min}$ and reperfusion for $2 \mathrm{~h}$ ); ischemia reperfusion $+\mathrm{miR}$ 126 mimic group $(\mathrm{N}=16,7$ days after miR-126 mimic transfection, myocardial ischemia for $30 \mathrm{~min}$ and reperfusion for $2 \mathrm{~h}$ ); ischemia reperfusion + miR-126 inhibitor group $(\mathrm{N}=16,7$ days after miR126 inhibitor transfection, myocardial ischemia for $30 \mathrm{~min}$ and reperfusion for $2 \mathrm{~h}$. Rat lentivirus particles were provided by the Shanghai Hanbio Co., Ltd. All animal experiments were approved by the animal ethics committee of Jiangxi Provincial People's Hospital. 
The ischemia reperfusion procedure was carried out on anesthetized animals (ip injection of $50 \mathrm{mg} / \mathrm{kg}$ sodium pentobarbital). The rats underwent a tracheotomy and received artificial respiration (breathing frequency: 60 times/min). A ligature of $1 \mathrm{~mm}$ was made near the lower edge of the left atrium and was maintained for $10 \mathrm{~min}$. The left anterior descending coronary artery was ligated with a small silicone tube between the blood vessels and the ligature. The ligature was relaxed after 30 min of myocardial ischemia, after which the myocardia were reperfused for $120 \mathrm{~min}$.

\section{Infarction area determination}

After reperfusion, $3 \mathrm{~mL}$ 2\% (w/v) Evans blue was injected into to the left anterior descending coronary artery in order to visualize ischemic and non-ischemic areas. After washing, cooling, and sectioning, the left ventricle slice was stained with $1 \%$ triphenyltetrazolium chloride (TTC; $37^{\circ} \mathrm{C}, 15$ $\mathrm{min}$ ) to allow for infarction tissue to be identified. The infarction area determination was carried out according to methods described by Ji and colleagues (Ji et al., 2004).

\section{TUNEL assay}

After dewaxing and rehydration of rat myocardial cells by xylene and ethanol, the cells were treated with $0.1 \%$ Triton X-100 and $0.1 \%$ sodium citrate for 15 min. A TUNEL (Roche, Indianapolis, IN, USA) assay was carried out on the cells to assess cell apoptosis. The tissue sections were allowed to react with terminal deoxynucleotidyl transferase $(\mathrm{TdT})$ at $37^{\circ} \mathrm{C}$ for $1 \mathrm{~h}$, after which DAPI was used to stain the nuclei. TUNEL-positive cells were identified by confocal microscopy (Zeiss, LSM 710).

\section{Statistical analysis}

All statistical analyses were performed using SPSS18.0 software (Chicago, IL) and numerical data are presented as means and standard deviation (SD). Differences between multiple groups were analyzed using one-way ANOVA and LSD $t$-test. Differences with $\mathrm{P}<0.05$ were considered significant.

\section{RESULTS}

\section{miR-126 is downregulated in myocardial cells subjected to ischemia-reperfusion injury}

Rat myocardial cells harvested after the induction of ischemia-reperfusion were shown by real-time PCR to have significantly decreased miR-126 levels $(P<0.05)$ (Figure 1$)$.

\section{miR-126 reduces myocardial cell apoptosis caused by ischemia-reperfusion injury}

Rat myocardial cells were transfected with miR-126 mimic and inhibitor, after which cell death was assessed by flow cytometry. This assessment revealed that, compared with the no ischemia-reperfusion injury group, the miR-126 mimic significantly increased myocardial cell apoptosis $(P<0.05)$, while the miR-126 inhibitor significantly reduced myocardial cell apoptosis $(P$ $<0.05$ ) (Figure 2). 


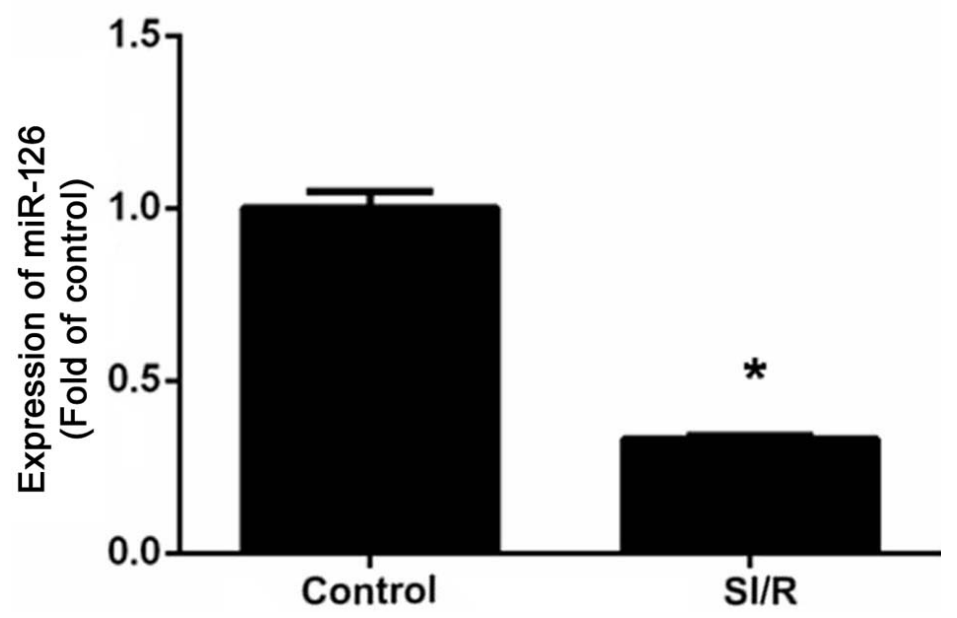

Figure 1. In vitro miR-126 expression in myocardial ischemia-reperfusion (SI/R) injury.

A

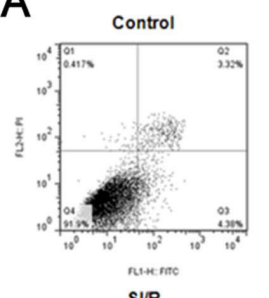

SUR

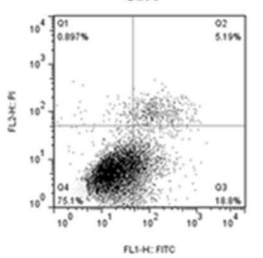

Control+miR-126 mimic

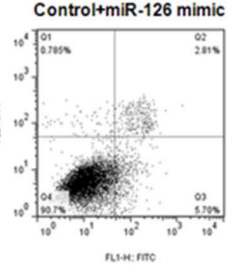

SIR+miR-126 mimic

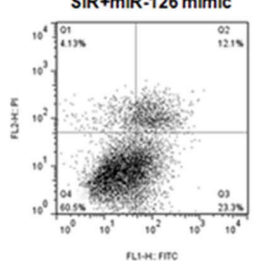

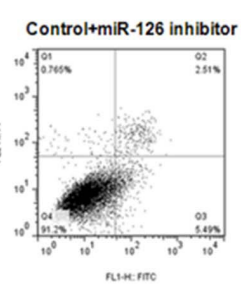

SIR+miR-126 inhibitor

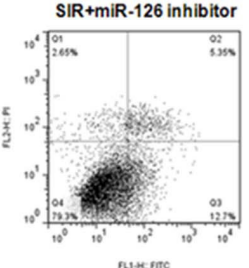

B

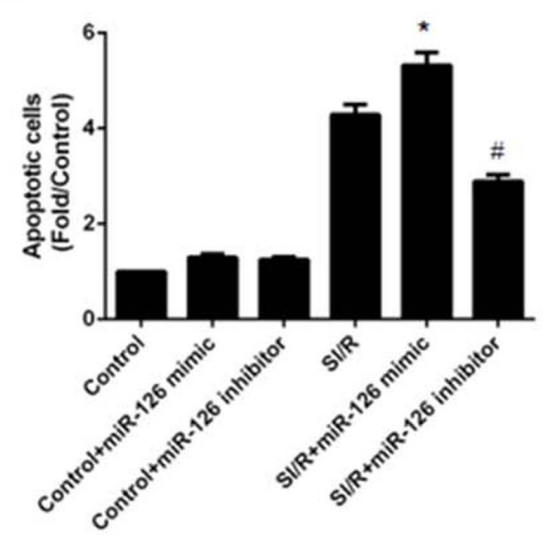

Figure 2. Reduction in ischemia-reperfusion (SI/R) injury-induced myocardial cell apoptosis by miR-126 inhibitor.

\section{miR-126 regulation affects caspase-3 expression in myocardial cells following ischemia-reperfusion}

Rat myocardial cells were transfected with miR-126 mimic and inhibitor, after which ischemia-reperfusion injury was induced. Western blot analysis revealed that the miR-126 mimic significantly increased caspase 3 protein expression $(P<0.05)$, whereas the miR-126 inhibitor reduced caspase 3 protein expression $(P<0.05)$ (Figure 3 ).

\section{miR-126 regulation affects rat myocardial ischemia-reperfusion infarction size}

Lentivirus miR-126 mimic and inhibitor were injected into rat myocardia to establish an 
ischemia-reperfusion injury model, after which the TTC method was used to identify the myocardial tissue infarction area. The results of this assessment (Figure 4) show that, compared with the control group of I/R injury, miR-126 mimic lentivirus-injected animals exhibited markedly larger infarction areas $(P<0.05)$, and miR-126 inhibitor lentivirus-injected animals exhibited significantly smaller infarction areas $(P<0.05)$.

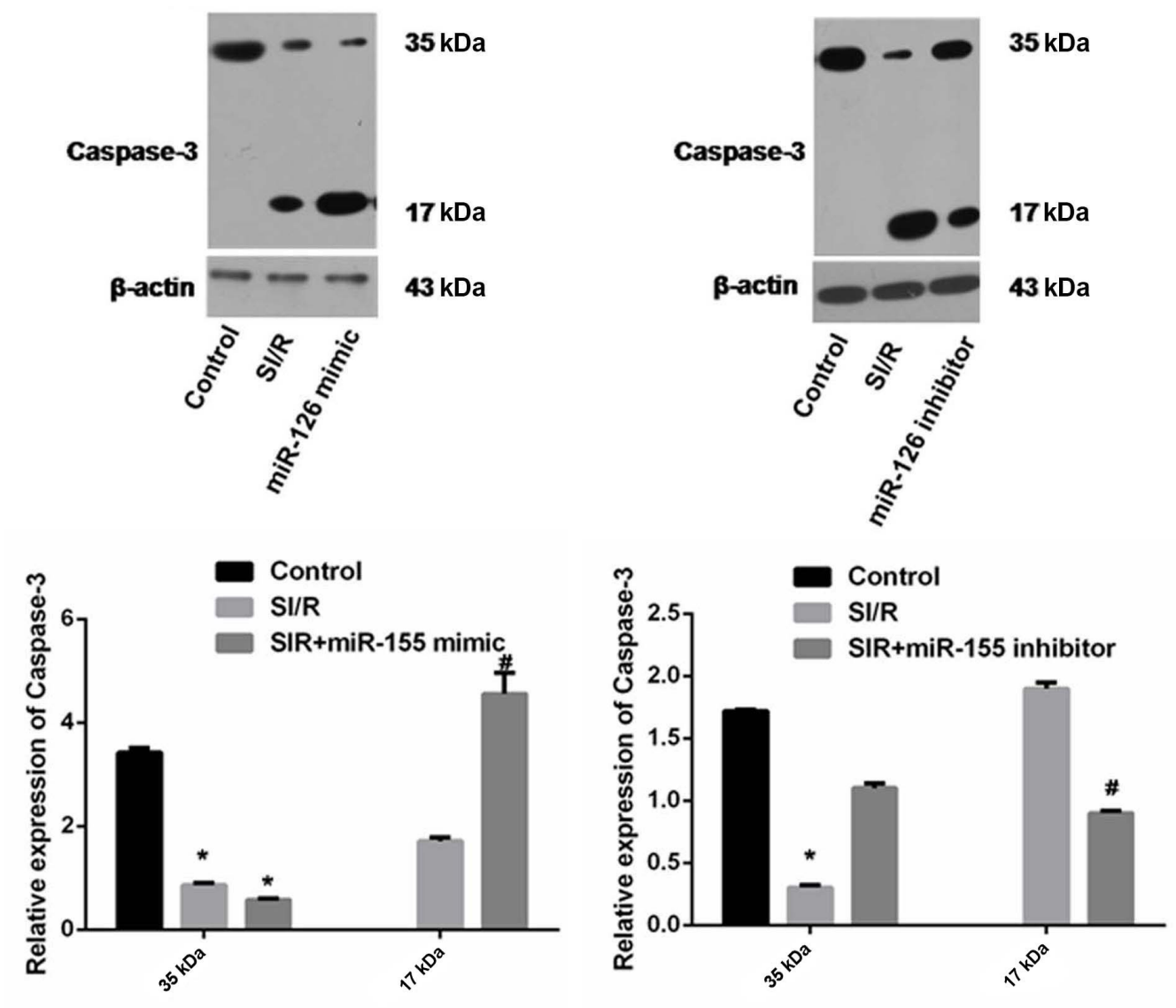

Figure 3. Effects of miR-126 overexpression and inhibition on caspase- 3 expression in myocardial cells following ischemia-reperfusion. ${ }^{*} P<0.05$ compared with control; ${ }^{P} P<0.05$ compared with reperfusion injury group.

\section{miR-126 regulation affects myocardial cell apoptosis in rat ischemia-reperfusion injury}

Lentivirus miR-126 inhibitor and mimic were introduced to rat myocardia, after which ischemia-reperfusion injury was induced. The TUNEL assay was used to assess myocardial cell apoptosis, and revealed that the miR-126 mimic lentivirus markedly increased cell apoptosis in myocardial tissue $(P<0.05)$, while the miR-126 inhibitor lentivirus significantly reduced the cell apoptosis rate $(P<0.05)$ (Figure 5$)$. 


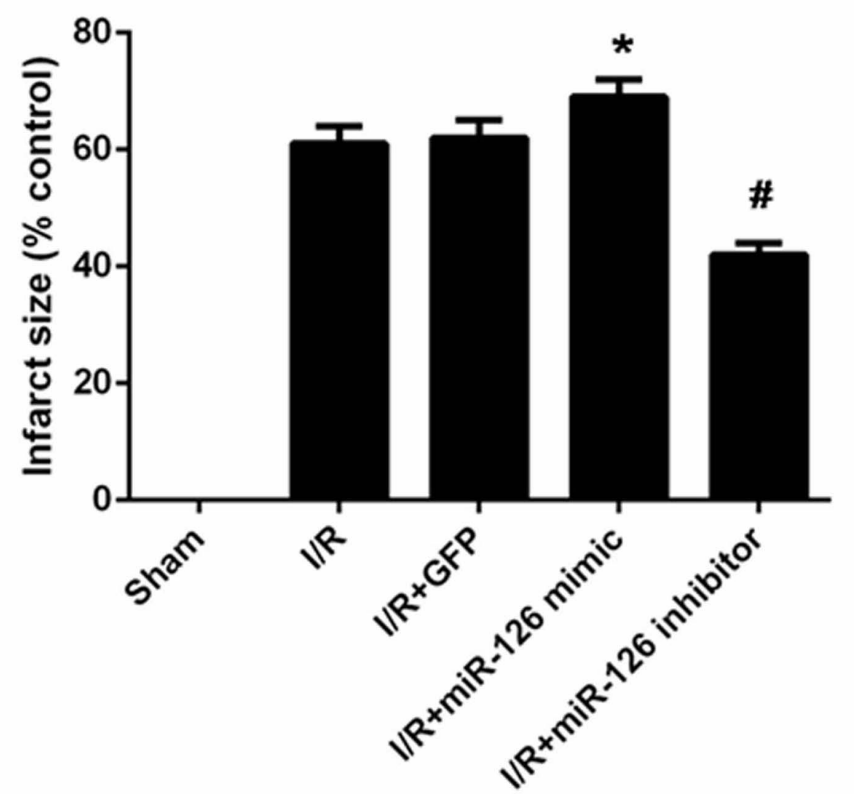

Figure 4. Effects of miR-126 overexpression and suppression on rat myocardial ischemia-reperfusion infarction area size. ${ }^{*} \mathrm{P}<0.05$ compared with I/R group; ${ }^{*} \mathrm{P}<0.05$ compared with $\mathrm{I} / \mathrm{R}+\mathrm{miR}-126$ mimic.

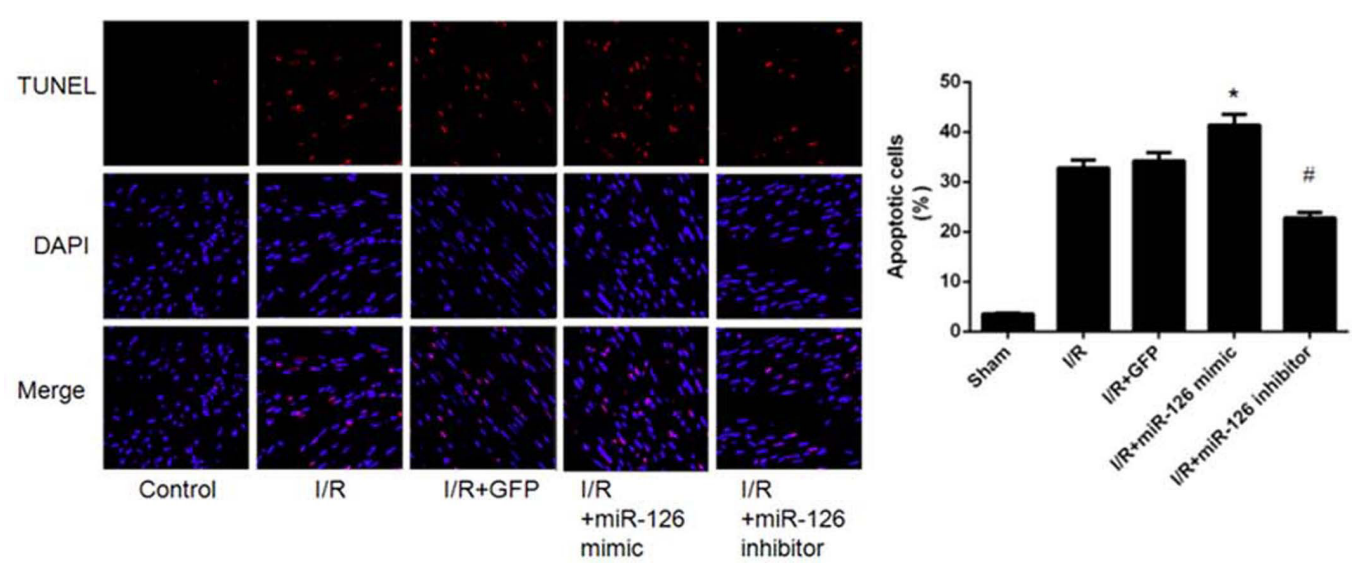

Figure 5. Effects of miR-126 overexpression and suppression on myocardial cell apoptosis in rat ischemia-reperfusion. ${ }^{*} \mathrm{P}<0.05$ compared with $\mathrm{I} / \mathrm{R}$ group; ${ }^{*} \mathrm{P}<0.05$ compared with $\mathrm{I} / \mathrm{R}+\mathrm{miR}-126$ mimic.

\section{DISCUSSION}

Ischemia-reperfusion injury has become an important focus in the field of cardiovascular research (Freeman et al., 2014; Penna et al., 2014). Ischemia-reperfusion injury has been associated with oxygen free radicals, vascular endothelial injury, and myocardial apoptosis (Chen et al., 2014) and miRNAs have also been shown to play a role in the regulation of myocardial cells (Zhang et al., 2014). Therefore, in this study, the role of miR-126 in myocardial ischemia- 
reperfusion injury was assessed in vitro and in vivo.

miR-126 expression was found to be significantly increased in the myocardial ischemia reperfusion cell model, and transfected with miR-126 mimic or inhibitor affected the myocardial cells apoptosis rate. Western blot analysis furthermore showed that caspase 3 expression was also affected by transfection with miR-126. Rat models of myocardial ischemia-reperfusion injury were injected with miR-126 mimic and inhibitor lentivirus, after which increases and decreases in the myocardial tissue infarction area and myocardial cell apoptosis were observed for the inhibitor and the mimic, respectively. These findings are the first to demonstrate a role for miR-126 in myocardial ischemia-reperfusion injury.

In a study by Long and colleagues it was shown that miR-126 is mainly expressed in the endothelial cells and negatively regulates angiogenesis. The findings of their study suggest that miR-126 plays an important role in vascular integrity and angiogenesis (Long et al., 2013). After the occurrence of myocardial ischemia, myocardial vascular endothelial cells are damaged, which activates the body's protective mechanism and the ability to form new blood vessels. In this context, miR-126 downregulation benefits angiogenesis and myocardium recovery, which is in accordance with findings reported by Banerjee (Banerjee et al., 2013) and Li (Li et al., 2013) regarding tumors. We furthermore found that the inhibition of miR-126 may result in a decline in myocardial cell apoptosis in vitro and in vivo.

At present, the mechanism of cell apoptosis in ischemia-reperfusion injury is still not clear. Jiang and colleagues (Jiang et al., 2014), however, suggested recently that miR-126 may participate in myocardial cell apoptosis via heat shock protein 70 . The target genes of miR-126 were not confirmed in this study and require further investigation to allow for the exact mechanism of miR-126 in the regulation of myocardial cell apoptosis to be elucidated.

In conclusion, miR-126 expression was found to be affected by myocardial ischemia reperfusion injury. Inhibition of miR-126 was furthermore found to effectively reduce myocardial cell apoptosis. The findings reported here represent the identification of a novel molecule in myocardial ischemia-reperfusion injury and thus provide a possible drug target for clinical treatment.

\section{REFERENCES}

Alexandre J, Schiariti M, Rouet R and Puddu PE (2013). Rabbit ventricular myocardium undergoing simulated ischemia and reperfusion in a double compartment tissue bath: a model to investigate both antiarrhythmic and arrhythmogenic likelihood. Int. J. Physiol. Pathophysiol. Pharmacol. 5: 52-60.

Banerjee N, Kim H, Talcott S and Mertens-Talcott S (2013). Pomegranate polyphenolics suppressed azoxymethane-induced colorectal aberrant crypt foci and inflammation: possible role of miR-126/VCAM-1 and miR-126/PI3K/AKT/mTOR. Carcinogenesis 34: 2814-2822.

Catalyurek H, Oktay G, Guzeloglu M, Cavdar Z, et al. (2008). Insulin-blood cardioplegia decreases matrix metalloproteinase activity in ischaemia-reperfusion injury during coronary artery bypass surgery. J. Int. Med. Res. 36: 551-558.

Cayan S, Saylam B, Tiftik N, Ünal ND, et al. (2014). Rho-kinase levels in testicular ischemia-reperfusion injury and effects of its inhibitor, Y-27632, on oxidative stress, spermatogenesis, and apoptosis. Urology 83: 675 e13-e18.

Chang KP, Lee HC, Huang SH, Lee SS, et al. (2012). MicroRNA signatures in ischemia-reperfusion injury. Ann. Plast. Surg. 69: 668-671.

Chen CL, Yang J, James IO, Zhang HY, et al. (2014). Heparin-binding epidermal growth factor-like growth factor restores Wnt/ beta-catenin signaling in intestinal stem cells exposed to ischemia/reperfusion injury. Surgery 155: 1069-1080.

Chen Y, Zhou P, Yan H, Zhao H, et al. (2013). Impact of selective infarct-related artery infusion of tirofiban on myocardial reperfusion and bleeding complications in patients with acute myocardial infarction: the SUIT-AMI trial. J. Invasive Cardiol. 25: 376-382.

Cong B, Zhu X, Cao B, Xiao J, et al. (2013). Estrogens protect myocardium against ischemia/reperfusion insult by up-regulation of $\mathrm{CRH}$ receptor type 2 in female rats. Int. J. Cardiol. 168: 4755-4760. 
Corsten MF, Dennert R, Jochems S, Kuznetsova T, et al. (2010). Circulating MicroRNA-208b and MicroRNA-499 reflect myocardial damage in cardiovascular disease. Circ. Cardiovasc. Genet. 3: 499-506.

Ekhterae D, Lin Z, Lundberg MS, Crow MT, et al. (1999). ARC inhibits cytochrome c release from mitochondria and protects against hypoxia-induced apoptosis in heart-derived H9c2 cells. Circ. Res. 85: e70-e77.

Freeman CM, Quillin RC 3rd, Wilson GC, Nojima H, et al. (2014). Characterization of microparticles after hepatic ischemiareperfusion injury. PLoS One. 9: e97945.

Guo C, Sah JF, Beard L, Wilson JK, et al. (2008). The noncoding RNA, miR-126, suppresses the growth of neoplastic cells by targeting phosphatidylinositol 3-kinase signaling and is frequently lost in colon cancers. Genes Chromosomes Cancer 47: 939-46.

Hadi NR, Yusif, FG, Yousif M and Jaen KK (2014). Both castration and goserelin acetate ameliorate myocardial ischemia reperfusion injury and apoptosis in male rats. ISRN Pharmacol. 2014: 206951.

Ji ES, Yue H, Wu YM and He RR (2004). Effects of phytoestrogen genistein on myocardial ischemia/reperfusion injury and apoptosis in rabbits. Acta Pharmacol. Sin. 25: 306-312.

Jiang C, Ji N, Luo G, Ni S, et al. (2014). The effects and mechanism of miR-92a and miR-126 on myocardial apoptosis in mouse ischemia-reperfusion model. Cell Biochem Biophys. 70: 1901-6.

$\mathrm{Li}$ Z, Li N, Wu M, Li X, et al. (2013). Expression of miR-126 suppresses migration and invasion of colon cancer cells by targeting CXCR4. Mol. Cell Biochem. 381: 233-242.

Liu X, He S, Skogerbø G, Gong F, et al. (2012). Integrated sequence-structure motifs suffice to identify microRNA precursors. PLoS One 7: e32797.

Liu ZB, Fu XH, Wei G, and Gao JL (2014). Cytochrome c release in acute myocardial infarction predicts poor prognosis and myocardial reperfusion on contrast-enhanced magnetic resonance imaging. Coron Artery Dis. 25: 66-72.

Long G, Wang F, Li H, Yin Z, et al. (2013). Circulating miR-30a, miR-126 and let-7b as biomarker for ischemic stroke in humans. BMC Neurol. 13: 178.

Medina HM, Cannon CP, Fonarow GC, Grau-Sepulveda MV, et al. (2012). Reperfusion strategies and quality of care in 5339 patients age 80 years or older presenting with ST-elevation myocardial infarction: analysis from get with the guidelinescoronary artery disease. Clin. Cardiol. 35: 632-640.

Penna C, Brancaccio M, Tullio F, Rubinetto C, et al. (2014). Overexpression of the muscle-specific protein, melusin, protects from cardiac ischemia/reperfusion injury. Basic Res. Cardiol. 109: 418.

Pirhan O, Ozcelik F, Demir B, Karakus G, et al. (2013). Correlation of myocardial performance index assessed by different echocardiographic methods in patients with acute myocard infarction receiving different reperfusion treatment. Minerva Med. 104: 593-604.

Produit-Zengaffinen N, Pournaras CJ and Schorderet DF (2014). Autophagy induction does not protect retina against apoptosis in ischemia/reperfusion model. Adv. Exp. Med. Biol. 801: 677-683.

Qiao X, Xu J, Yang QJ, Du Y, et al. (2013). Transient acidosis during early reperfusion attenuates myocardium ischemia reperfusion injury via PI3k-Akt-eNOS signaling pathway. Oxid Med Cell Longev. 2013: 126083.

Quarles KA, Sahu D, Havens MA, Forsyth ER, et al. (2013). Ensemble analysis of primary microRNA structure reveals an extensive capacity to deform near the Drosha cleavage site. Biochemistry 52: 795-807.

Shapiro MD, Bagley J, Latz J, Godwin JG, et al. (2011). MicroRNA expression data reveals a signature of kidney damage following ischemia reperfusion injury. PLoS One 6: e23011.

Tomasetti M, Nocchi L, Staffolani S, Manzella N, et al. (2014). MicroRNA-126 suppresses mesothelioma malignancy by targeting IRS1 and interfering with the mitochondrial function. Antioxid. Redox Signal. 21: 2109-2125.

Tu Y, Wan L, Fan Y, Wang K, et al. (2013). Ischemic postconditioning-mediated miRNA-21 protects against cardiac ischemia/ reperfusion injury via PTEN/Akt pathway. PLoS One 8: e75872.

Yaghoubi A, Danaee S, Imani S, Sheikhalizadeh M, et al. (2011). Effect of citrate phosphate dextrose solution on reperfusion injury in coronary artery bypass surgical patients undergoing cardiopulmonary bypass. J. Cardiovasc. Thorac. Res. 3: 123-127.

Zhang Z, Zhang H, Li H, Chen X, et al. (2014). Selective expression of tumor necrosis factor-related apoptosis-inducing ligand mediated by microRNA suppresses renal carcinoma growth. Mol. Cell Biochem. 392: 125-134. 